FEM-2019048

Verso: Front. Eng. Manag.

Recto: Conrad BOTON et al. Home-made mobile technologies on construction sites

https://doi.org/10.1007/s42524-019-0065-9

RESEARCH ARTICLE

Conrad BOTON, Éric ST-PIERRE, Gabriel LEFEBVRE

\title{
Should medium-sized contractors still implement home-made information technologies on construction sites?
}

(C) Higher Education Press 2020

\begin{abstract}
Despite the increasing dissemination of the Building Information Modeling (BIM) approach, many firms are still far from BIM and are only starting to adopt information technologies. We conducted an ethnographic action research with an industrial partner that implements information technologies on its construction sites, with a strong preference for home-made applications. Results show that the development of such applications faces difficulties and that multiple iterations and versions are necessary before achieving the expected goals because the required competencies are lacking among internal development teams. Moreover, initial needs evolve considerably throughout the application's development and evaluation process. However, notwithstanding the difficulties encountered during the different application development phases, the industrial partner believes the decision to be worthwhile because of the desire to have internal control over the project data and the need to consider certain specificities related to business processes.
\end{abstract}

Keywords small- and medium-sized enterprises, mobile computing, mobile cloud computing, information technology for construction, construction site, ethnographic action research

\section{Introduction}

In the last decades, the construction industry has undergone a digital transition, manifested by the growing role of information technology (IT) in practices (Kubicki and Boton 2014; Samuelson and Björk, 2014). In this context, the Building Information Modeling (BIM) approach appears to occupy an important place, and several works have shown its added value and its uses at different stages of a construction project (Sacks et al., 2018). In addition, BIM is increasingly being disseminated in architectural, engineering, and construction firms (Jung and Lee, 2015; Kassem and Succar, 2017). Nevertheless, several firms are still far from using BIM and are only starting to adopt information technologies (Kubicki and Boton, 2014). The situation with general contractors and subcontractors is challenging because the adoption of IT in the office must be accompanied by a similar adoption of mobile technologies on construction sites to optimize information exchange between the office and the construction site (Ahn et al., 2016).

The adoption of mobile technologies on-site poses significant challenges because of the peculiarities and particular requirements of construction site work. Thus, the research on the subject is often based on generic solutions (Chen and Kamara, 2008; Anumba and Wang, 2012) and is limited to the study of related issues (Chen and Kamara, 2011; Yeh et al., 2012), the link with BIM and the ability of site actors to adapt their work processes to these tools (Kimoto et al., 2005; Davies and Harty, 2013; Park et al., 2016; Svalestuen et al., 2017), and the study of perceived added value (Marsh and Flanagan, 2000; Bowden et al., 2006; Omar and Nehdi, 2016; 
Forcada et al., 2017; Hasan et al., 2019). Very few studies (Svalestuen et al., 2017) have explored the issue using a participatory approach to identify the real needs of firms, their ability to meet their own needs with their in-house resources, and the issues associated with their decisions. The present research was conducted at the premises of an industrial partner that implements information technologies on its construction sites, with a strong preference for home-made applications. The objective of the research is to understand the internal dynamic proceeding from the initial situation of the firm and the needs expressed toward a solution adapted to the realities of its construction sites. In specific, it aims to provide an overview of the process while identifying the main associated issues to determine whether investing in the development of home-made information technologies rather than using commercial software is worthwhile for a typical medium-sized contractor.

The article is organized into four main parts. The first part presents related works, including recent similar works. The second part introduces the methodology used in the research, based on ethnographic action research. The third part presents the results obtained in the framework of the context and the needs, the choice of the device, the development of a mobile application, and the evaluation of the proposed solutions. These results are discussed in the fourth part to highlight how they fare as compared to recent similar works.

\section{Related works}

In this section, we present related research works, including those pertaining to IT in the construction industry, mobile computing for construction sites, and recent trends.

\subsection{IT in the construction industry}

\subsubsection{Peculiarities of the construction industry}

The construction sector is a major sector of national economies, where it generally has a major social, economic, and ecological impact (Sunke, 2009). The life cycle of a built facility can be broken down into several phases. Basing from previous works (Neufert, 2002; Boton, 2013; Boton et al., 2018), we can classify these phases into the pre-project phase (assembly, feasibility studies, and search for funding), the pre-construction phase (design, allotment, allocation of lots, and site preparation), the construction phase (construction of the structure), and the post-construction phase (reception, operation, rehabilitation, or demolition).

Several actors play different roles in these phases. These stakeholders include the owners, to the extent that they define the needs and the program, finance the project, and recruit the project team at the beginning of a construction project. Project managers are usually architects responsible for designing the facility and proposing an "architectural, technical, and economic response to the client's program" (Kubicki, 2006). The team also includes engineers, consultants, and other specialists, depending on the type and requirements of the project. Contractors and subcontractors are responsible for carrying out the construction work, which occurs on a construction site, involving different trades (earthworks, carcass work, framing, covering, insulation, painting, etc.), and working in contractual configurations that may vary (design-bid-build, design-build, integrated project delivery, etc.).

Construction projects share peculiarities that make them stand out from conventional projects encountered in certain industrial fields, such as aeronautics or automobiles. While Patrick (2003) emphasizes the fact that in both construction and other cases, we are looking at a similar fabrication of a product using appropriate resources (materials, equipment, labor), and the product is manufactured using a specific process. According to Austin et al. (2001), construction projects are distinguished by three main points: 1) they are always unique activities with distinct objectives; 2) resources are used on each construction project in a single configuration, which means each project is specific and different; and 3) the environment of a construction project is always different, for example, certain circumstances of the project (legal jurisdictions, market conditions, management mechanisms, etc.) can change often. Baccarini (1996) adds to these points the existence and commitment of several diverse and separate organizations, requiring that temporary organizational structures and contractual forms that vary per project to be set up. Sunke (2009) 
focuses on eight properties to consider: Changing production sites, spatial constraints, dependence on seasons and weather conditions, construction- and country-specific legislation (particularly, environmental legislation), a time/resource compromise, simultaneity of several projects, uncertain planning environment, and a combination of multiple objectives (time, cost, quality, and upgrading of resources).

One of the concepts commonly used to characterize the construction industry is fragmentation. Howard et al. (1989) distinguished vertical fragmentation from horizontal fragmentation. Vertical fragmentation relates to the fact that a construction project is subdivided into several short phases; in horizontal fragmentation, multiple different specialists intervene during the same phase. Combining vertical and horizontal fragmentation produces small specialized firms operating in small local markets. Although such specialization is expected to contribute to flexibility and generate benefits for the sector, it also generates additional costs in terms of fragmented decision making among managers, designers, tradespersons, and other users (Alashwal and Fong, 2015; Howard et al., 1989). IT is considered a solution to reduce fragmentation in the industry (Howard et al., 1989), and, in the context of BIM, Turk showed that increased fragmentation brought in more specialized knowledge, and the greater knowledge resulted in better quality and higher efficiency (Turk, 2016).

\subsubsection{Information technology in construction}

Two main flows appear in the production chain of a construction activity, as proposed by Sunke (2009): The flow of material and the flow of information, with strong interactions between the two. These flows correspond to the two major sub-processes identified by Björk (1999), namely, information and hardware. The material sub-process is the more tangible of the two but needs the information sub-process, considering that information is the third fundamental component of sociotechnical systems after material and energy (Ahmad et al., 1995). While the construction sector is considered resistant to new information and communication technologies, it is becoming more and more open to adopting new systems.

IT can be defined as "the use of electronic machines and programs for the processing, storage, transfer and presentation of information" (Björk, 1999). The formats commonly used to represent information in construction are text documents, 2D and 3D drawings, schedules in bar chart and other formats, various diagrams, charts, and tables, and so on. IT has dramatically improved how interdisciplinary construction project work is described and documented (Fischer and Kunz, 2004). Ahmad et al. (1995) summarized the characteristics of the construction industry, arguing that processes are dynamic and based on the complex interrelationships between the various participating entities, wherein teamwork is necessary to successfully construct a facility, and operations are people intensive and cannot be completely automated or mechanized. The solutions to construction project problems are highly dependent on the experience and judgment of professionals and involve uncertainties. These barriers to applying IT in architecture, engineering, and construction (AEC) lead to a challenging and interesting problem in producing an inventory of the use and impact of IT on the industry. Studies have been conducted on the construction industries in Canada (Rivard, 2000), China (Futcher and Rowlinson, 1999), Denmark, Sweden, Finland (Howard et al., 1998; Samuelson, 2008), Luxembourg (Kubicki and Boton, 2014), and other countries to evaluate the use and impact of IT on construction.

These studies found that the construction industry lags behind other industries, such as automotive and aerospace, in the use of IT. Debate on the subject centers on the premise that while demand actually exists, current technology-based solutions proposed to the industry are not adapted enough to support current needs (Bowden et al., 2006; Hartmann et al., 2012; Boton et al., 2013). According to Bowden et al. (2006), those involved in the development of such systems focus on what is technically exciting and on what presents great challenges rather than on the real needs and issues facing construction companies. Several other factors could also contribute to delays in the use of IT, which can be considered as barriers to adoption. The factors can be summarized as follows: Lack of leadership, resistance to change, fragmentation of the industry, difficulty of use, and lack of internal competencies.

IT is normally used to improve data transfers and productivity through less time wasting, less duplication of information, better communication, and better accuracy (Orihuela et al., 2016). For 
general contractors and subcontractors, a significant challenge in adopting IT lies in the use of mobile technologies to ensure the continuity of information between the office and the site. Recently, Alsafouri and Ayer (2018) have proposed a review of the literature on the information and communications technology (ICT) implemented to facilitate the flow of information between virtual models and construction project sites. 3D Laser Scanning, Radio Frequency Identification, and Mobile Computing are the most frequently used ICTs in the analyzed publications (Alsafouri and Ayer, 2018). Due to the dynamic nature of the production work in construction, "there is a need to integrate advances in mobile computing in the work environment to provide user-friendly and mobile access to construction product and production process" (Aziz et al., 2017).

\subsection{Mobile computing for construction sites}

\subsubsection{Mobile computing in construction}

According to Rebolj and Menzel (2004), the AEC industry is a good field for the implementation of mobile computing because of its specific characteristics. They defined mobile computing through three main components: 1) computers usable while being in motion, excluding conventional notebooks, 2) networks with enough bandwidth accessible while in motion, and 3) mobile applications to support personalization and context sensitivity. Mobile IT, which includes tablet PCs, all kinds of pocket computers, and wearable computers (Rebolj and Menzel, 2004), enables workers to roam seamlessly with computing and communication functionalities in an uninterrupted way (Anumba and Wang, 2012). Its deployment has been enabled in different industries by the development of "affordable mobile technologies", making it common for mobile users to capture, store and reuse information (Bowden et al., 2006). Rebolj and Menzel (2004) argued that mobile computing is not just another IT but an important factor in construction, with the potential to increase the effective use of IT in an integrated and holistic way (Chen and Kamara, 2011). Indeed, mobile computing can extend the boundary of information systems from site offices to actual work sites and ensure real-time data flow to and from construction work sites (Chen and Kamara, 2011).

Chen and Kamara (2011) proposed a framework for the use of mobile computing for managing information on construction sites. The framework identified six primary components, including three dependent factors (mobile computer, mobile application, and wireless network) and three independent factors (user, construction information, and construction site). The dependent factors are the fundamentals of the mobile computing concept, whereas the independent factors determine the particular context in which the mobile computing is used. Anumba and Wang (2012) categorized the characteristics of mobile computing device capabilities into technical functionality (including mobile communication, limited communication, memory and processing capacities, time-critical services, and personal touch), portability (including different weights, sizes, input mechanisms, displays, storage capacities, and performance), and situational awareness (including location and identity awareness).

However, they noted several constraints that make mobile computing different from conventional IT in construction (Anumba and Wang, 2012): 1) mobile information technologies are more constrained in terms of resource as compared with conventional IT systems; 2) mobile devices are more vulnerable because they can be exposed to rough and unsafe environments; and 3) the connectivity of mobile devices can show limited stability in terms of latency and bandwidth, resulting in frequent disconnection, especially in concrete-framed buildings. Mobile cloud computing usually has the potential to overcome these constraints.

\subsubsection{Mobile cloud computing}

Nowadays, technology has evolved dramatically, and most of the preceding issues are no longer as pertinent. Mobile cloud computing has considerably increased mobile device computing resources (Silverio et al., 2017). It combines cloud-based storage and application processing services to improve compute-intensive applications and off-device storage on mobile devices. This computing can implement multiple strategies of augmentation (storage, screen, energy, and application processing) to alleviate the resource limitations of traditional mobile computing systems (Abolfazli et al., 2014; Silverio et al., 2017). It can thus greatly reduce the cost of mobile 
application development and execution while greatly extending the resources and services provided (Ahmed et al., 2015). Abolfazli et al. (2014) proposed a comparison between cloud-based computing and traditional resources, showing that cloud-based systems ensure high computational power, elasticity, user experience, reliability, security, and safety.

Silverio et al. (2017) have recently proposed a discussion on the main challenges related to mobile computing in the construction industry. Focusing on sustainable construction issues, they argued that mobile cloud computing can provide effective solutions for design-, material-, financial-, management-, and knowledge-related challenges. Bowden et al. (2006) speculated on the added value of mobile computing and identified several benefits for the industry: Reductions in construction time, cost, defects, accidents, and waste, and increase in productivity and predictability. More recently, Hasan et al. (2018) have presented an exploratory study that evaluated the impact of mobile IT on the productivity of construction projects. The study suggested that while mobile computing has undergone notable advances, its impact on productivity has been limited by differences in usage style and user attitude.

Anumba and Wang (2012) summarized the benefits of mobile computing in construction as follows: Nomadic construction workers can remain connected to the office while working at distributed locations; construction site workers can be more easily tracked and managed by project managers, and the progress of their work can be monitored in real time, allowing better management of the resources; distributed workers can benefit from effective communication systems; the right information and services can be delivered at the right time to the right person through context-awareness integration; collaboration can be considerably improved; the training provided to construction workers can benefit from tremendous opportunities; and safety and security can be improved through effective tracking of mobile construction personnel.

However, the integration of mobile computing on construction sites is still facing some important challenges.

\subsubsection{Recent related works}

Many recent studies have explored the question of the use of mobile computing on construction sites. Some of them have studied the use of mobile apps on construction sites, focusing on the added value brought to construction projects (Marsh and Flanagan, 2000; Bowden et al., 2006; Forcada et al., 2017; Hasan et al., 2019) or on extending the uses of BIM on construction sites, through mobile cloud computing (Davies and Harty, 2013; Park et al., 2016; Svalestuen et al., 2017).

With respect to the added value of mobile computing, Hasan et al. (2019) conducted a survey on the perceived productivity effect of mobile ICT on construction projects. The study only surveyed construction management professionals and suggested improvement in four main factors, namely, work flow and communication, project execution, data access, and construction defect management. Omar and Nehdi (2016) reviewed how mobile technologies can be integrated as data acquisition technologies for monitoring and tracking construction progress. The study showed that $\mathrm{AR}$ applications are promising for all types and sizes of projects and that lightweight mobiles and immersive AR systems are therefore recommended for field personnel due to the dynamic environment of construction fields. Forcada et al. (2017) explored how mobile applications can be used to assess building accessibility in smart cities. The study suggested that mobile computing can simplify and reduce the accessibility recording time, especially when standardized information is used to help in obtaining consistent data.

Regarding the link between BIM and mobile computing, a previous study observed an increase in use of BIMs in the construction process with BIM devices like BIM stations and tablets (Svalestuen et al., 2017). This observation can be explained by the fact that while BIM tools are well established in design phases, issues of adoption and implementation persist, particularly for on-site use of BIM tools in the construction phase (Davies and Harty, 2013). Park et al. (2016) proposed a construction site management application using mobile BIM associated with a location tracking technology. The proposed application aims at avoiding the inefficiency due to the separation between field management and digital project information, considering that project managers need to spend significant time and effort to find out useful project information (such as 
plans and specifications) for field management and communicate with construction participants in the site and offices. Svalestuen et al. (2017) used a survey of a major contractor in Norway to study the advantages and challenges associated with the use of such devices on construction sites. Results showed that the use of BIM is insufficient to improve "effectiveness" in construction projects, but that the use of BIM stations and tablets is equally important to continuously convey updated information for on-site jobs. Such devices present multiple advantages: Providing workers with a clear 3D-based understanding of the project; providing them with updated documentation; providing quantitative take-off directly on-site; providing tools to report task completion, errors, and quality issues; helping workers obtain direct measurements; establishing live video chat communication between the office and the construction site; reducing the risk of defects and errors; and decreasing printing and the use of paper-based documentation.

\section{Materials and methods}

In this section, we present the theoretical framework and the research approach used in the present study. It is based on the ethnographic action research principles. As demonstrated by Hartmann et al. (2009), ethnographic action research is well suited to support the development and implementation of information systems. In particular, it can be very helpful for the identification of specific problems on architecture, engineering, and construction projects to adapt information systems accordingly in close collaboration with the practitioners working on these projects.

\subsection{Theoretical framework}

\subsubsection{Definition of action research}

Action research is a research approach based on the belief that scientific data can be collected by acting first and foremost (Brydon-Miller et al., 2003). It is therefore the complete opposite of what is generally found in science, which calls first for an understanding, after which the theory is devised and then taken into practice. In action research, practice comes first, and then the theory is formed from the results obtained. The theory is thus based on actual solutions that have been proven in the field. Three important features of action research can be isolated (Roy and Prévost, 2013). First, it is performed with people rather than on people, and the people involved in the project are not subjects or objects of study. Secondly, it is included in the action by the desire to change things. Thus, change that requires action is immediately needed. Third, it takes a cyclical path. As a result, it allows continuous and flexible improvement. Therefore, establishing a precise timetable on the project to consider the scope of action or to have a final result is impossible because of the constant need for improvement (Roy and Prévost, 2013).

The cycle of action research is shown in Fig. 1. First, planning is important. Then, the stakeholders should be involved in the project, and the context and needs must be identified. Once the project is ready, it can be implemented, and some data can be collected to make an assessment and propose recommendations. Then, the project is adjusted, and a second data collection is executed, and so on. Therefore, the improvement is continuous and can be represented as a cyclical passage of information and recommendations.

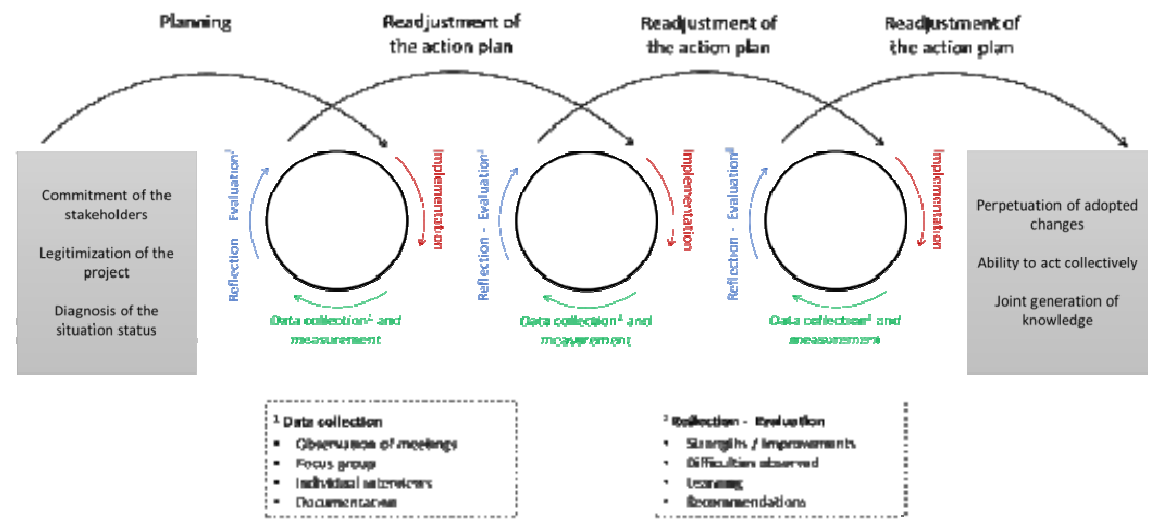

Fig. 1 Cycle of action research (adapted from Roy and Prévost (2013)). 


\subsubsection{Ethnographic action research for the implementation of IT in construction}

As previously mentioned, action research is a method that prioritizes practice and collaboration between practitioners and researchers throughout the project. However, it does not necessarily offer tools and techniques to allow a good understanding of the process. Ethnographic research proposes these tools and techniques for research. It was developed by anthropologists for the study of human culture, but in recent decades, it has been used as an instrument for observation of the implementation of technologies in systems. Ethnographic action research focuses on understanding the actions, thoughts, and feelings of professionals throughout their work day. The ethnographic action research approach proposed by Hartmann et al. (2009) allows the implementation of customized systems to meet the real needs of professionals. This type of research must draw information from several sources, such as existing and completed forms, interviews, observations, and reports. To be possible, it requires a good integration of the researcher into the project team. After all the information is gathered, a platform adapted to the needs can be determined and designed. With ethnographic action research, results must always be compared with initially identified needs.

Ethnographic action research is suited for the implementation of IT in the construction industry, characterized by its often-repetitive activities and many of its processes involving data entries (Hartmann et al., 2009). Indeed, it allows for a focused methodology for observing completed tasks and processes to understand business needs and to propose adapted solutions.

\subsection{Steps of our research}

In this research, we use the ethnographic action research principle explained above. The cycle of an ethnographic action research encompasses four complementary stages: Ethnographic observations, identification of work routines, information system development, and information system implementation on the project. In this section, we present how the theory has been implemented in the project. Observations, internal documentation reviews, and interviews are conducted to understand the business context and to identify the needs. The appropriate solutions are proposed according to these needs, and the mobile application has been developed and implemented in a pilot project for evaluation purposes.

\subsubsection{Ethnographic observations and identifying the work routines and needs}

The first step of the research was to understand the context, i.e., the current practices of the industrial partner. To this end, the principal researcher was involved in the firm and acted as a pivotal link among the upper management, the operational construction teams, and the IT department. Three data sources were used to ensure a good understanding of the practices and the needs: Observation, internal documentation review, and semi-directive interviews with the practitioners. Throughout the project duration, the main researcher had an office in the industrial partner's headquarter and was involved in its teams daily. Thus, the work practices were observed from different perspectives because he worked closely with both the IT department and the site crews. The observation helped to identify the ways of working various teams, the methods used, and the interactions and difficulties in certain situations. Particular emphasis has been placed on how to enter data for daily reports and additional worksheets and timesheets, and how these different documents are exchanged, processed, and stored. The industrial partner also allowed the principal investigator access to all internal documentations related to the project. An exhaustive review of the available documentation was conducted to understand the policies in place in the firm and the specific problems in information management and exchange. Finally, the researcher was able to take advantage of his presence to conduct informal discussions and semi-directed interviews with the construction site actors. For example, he tried to understand their actions when asked by the steel erectors or day laborers, or when faced with a lack of information on the construction site. 


\subsubsection{Choosing the appropriate hardware and developing the mobile application}

During this step, the main researcher was involved in the IT department team and worked closely with the internal IT specialists. He also served as the link between them and the construction site team to collect their feedback regarding the technological choices.

The choice and development of the technologies are important in the research. Because the industrial partner expressed a strong preference for in-house developed applications, the appropriate device was selected according to the requirements, and the work of the IT department was relied on for the development of the application.

The device selection was conducted in three steps. First, a list of requirements was established based on the previously identified needs. Considering these requirements, the researcher prepared a shortlist of devices that potentially meet the needs. Working with the internal developers, additional criteria were then used to preselect appropriate devices. Finally, discussions with upper management and the operational workers led to the choice of one device.

The application development was not that simple. Mock-ups of the final desired application were designed using Balsamiq, a rapid prototyping tool well known in programming, to ensure a common understanding of the expected results. Moreover, Unified Modeling Language (UML) diagrams were created and validated by the development team. Despite these efforts, multiple iterations were necessary before the final acceptable version was obtained. The main iterations corresponded to different preliminary versions of the application, which were tested and then abandoned due to technological issues or usability issues perceived by the workers.

\subsubsection{Implementing and evaluating the proposed information system}

The final stable version of the application was implemented through a pilot project for evaluation purposes. Both the device and the application were tested. The evaluation of the device was focused on its ability to be used effectively in extreme construction site conditions, especially in very cold weather. For its part, the evaluation of the application consisted in its use on a real construction site, after which feedback was collected from the foreman to evaluate how much it adapted to the identified needs. During this stage, the researcher made the trip to the site to test the device. He also took charge of collecting, directly from the construction site, the feedback from the practitioners.

\section{Main results}

To enable the reader to understand the issues and difficulties associated with the development of home-made solutions, the authors have chosen to document in detail all the steps of the process. This section presents the main results of the research project, including the context and the identified needs, the choice of the appropriate device, the development of the application, and the evaluation of the proposed technology through a pilot project.

\subsection{Context and needs}

\subsubsection{Context: Paper-based and time-consuming processes}

The industrial partner is a steel structure company that has been operating in the field for 40 years. It manufactures and installs steel frames and, in some cases, offers design services. When fully operational, the firm employs close to 150 people in four main environments: Factory, administration, drawing room, and construction site. Thus, the entire staff must have good management and communication to ensure continuity in information sharing and management.

Although cell phones and e-mails are widely used in the firm for communication, paper is still present and is certainly the most used tool for management by project managers. The same reality is observed in the many projects carried out by the company, which leads to considerable information duplication. Indeed, the use of paper remains a necessity because it allows forepersons and superintendents to do their work, including the management of incoming information from forepersons and clients (drawings, training plans, health and safety sheets, daily reports, inspection 
sheets, additional worksheets, etc.). Figure 2 shows the paper-based form used for the daily site report (French copy).

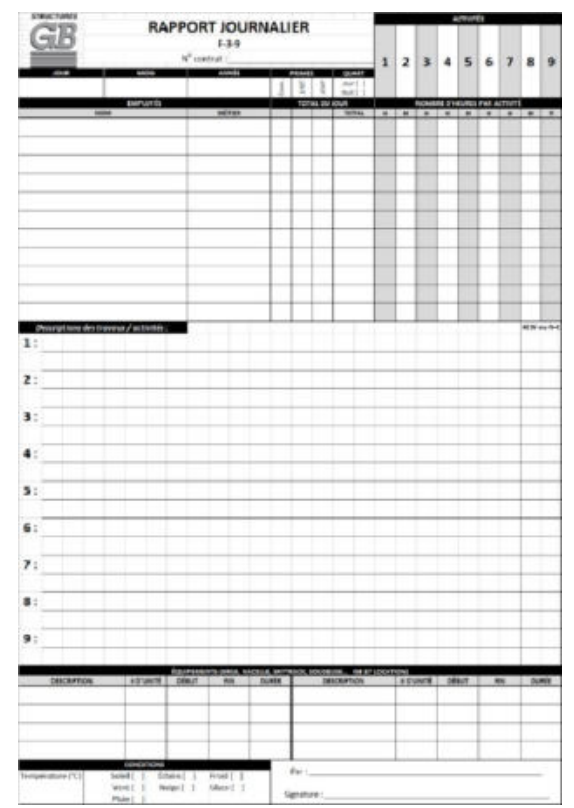

Fig. 2 Paper-based form used for the daily report (in French).

The most illustrative example of the importance of paper and the duplication of information is related to the management of the overtime worksheets. Once the overtime worksheets have been completed by the superintendent or the coordinator at the end of the day, the superintendent must sign them and send them to the client for approval and signature. After signed, he must scan and email them to the project manager. Then, he must place them in an envelope that will be mailed to the central office, which keeps the original copies in dedicated folders. Whenever things have to be verified by the project manager before the client's signature, the process time is considerably extended.

These long processes are time consuming. The fact that the paper is the main medium at the site imposes the need for several visits by the foreman to the trailer and site "shack". The information is thus centralized in only two places, one of which has only printed plans. This set-up leads to a loss of time, estimated at between 45 and $60 \mathrm{~min}$ per day for the back-and-forths of the forepersons. This is called "information search", according to one of the foremen interviewed. Following discussions with the site supervisors, even they believe that a lot of time is wasted trying to access the needed documentation and searching for information. A foreman described how he proceeds when he receives questions from steelworkers or day laborers: "Right now, when there is a problem, the steelworker calls me or signs me up to see him on the top floor of the building. When I arrive at the top and see the problem, I have to go down to the 'shack' to consult the appropriate drawings. I then take a close-up shot of the plans with my cell phone and I go back to the steelworker on the top floor to explain the correction to make. I'm wasting a lot of time. In addition, I take note of everything in my black notebook to transcribe a part in the daily report at the end of the day."

Regarding the action to take in case of a lack of information on the construction site, the foreman answered: "I need to go back and forth between the yard and the management trailer so that either the coordinator or the superintendent will print the design drawing... There is a big waste of time due to the search for information."

Forepersons must approach the superintendent or the coordinator at the construction trailer, which is not necessarily close by. This event can easily lead to considerable loss of time and information. These issues in the construction process may be related to a lack of access to information and communication. 


\subsubsection{IT needs}

Following the study of the internal documentation, observations of real-life experiences in the field, and interviews with stakeholders, the most important needs were identified to understand the current processes of the company and the review of the literature. These needs were related to information sharing and communication, access to information and simplification of existing processes, the mobility and the durability of tools, and increased productivity. Specific needs, such as those relating to the technology choice (hardware and software), helped guide the final decision and meet the needs of the company. Information on these needs was obtained from both the practitioners and the in-house programmers. Particular attention was paid to the work environment and the harsh construction site conditions.

The selected hardware then had to meet the durability, strength, and versatility requirements, in addition to conventional requirements, such as known operating system, performance, and storage capacity. These requirements can be summarized as follows:

- Durability: A product should not age considerably in time and should always be in use, that is, it is designed to last and receive updates that improve its performance.

- Resistance: A product should be resistant to shocks and bad weather/temperatures. Construction shelves tend to fall to the ground, come in contact with dust and water, and be subject to inclement weather. In addition, it must be able to cope with an operating temperature below zero and to meet the sometimes-extreme conditions of construction sites.

- Versatility: A product should be capable of allowing the addition of technological modules to meet other needs.

- Operating System: An operating system should allow the development and the use of applications. The firm expresses a strong preference for solutions developed in-house, and the company's programmers are not familiar with application operating systems such as Android or IOS, but with Windows.

- Performance: These technologies need to manage a platform that uses large amounts of external data and perform synchronization. A technology that can support such requirements and that has already proven itself is important.

- Capacity: A product should be with sufficient space or memory to support storage needs. In addition, space must be added through removable memory.

\subsection{Choice of the appropriate device}

Ten tablets were selected and compared. The comparison elements are presented in Appendix A. To arrive at this comparison, the researcher gathered as much information as possible about each tablet, including technical datasheets, user manuals, presentation videos, and discussion forums. When necessary, electronic mails were exchanged with the manufacturers and, in some cases, phone calls were held with the vendors. Only three tablets most corresponding to the identified needs were chosen. The first was from Dell, the Latitude 7212 Rugged Extreme tablet. It meets all the needs presented above. However, it comes with a high price tag. The second is the FZ-G1 tablet, from Panasonic. Similar to the first tablet, it also meets all identified needs but has a slightly high price. The third one is the ET50/55 tablet from Zebra. Of the three products, it presents the most advantageous price for what it can offer as characteristics.

The best tablet was selected through discussions between the programmers and the practitioners. The third tablet mentioned above was discarded despite its advantageous price. Indeed, the performance and strength of the tablet could present risks related to its processor, its operating temperature, and its internal memory. Therefore, the choice came down to either the Dell or Panasonic tablet. Both are excellent candidates for the project. They are highly durable and present excellent water-, dust-, cold-, and fall-resistance. The versatility is observed in the range of options available to the user, in terms of applications, technological modules to integrate, and accessories. As requested, the Windows operating system is a must and allows programmers to proceed with their development. The two tablets also allow a choice of processors, thereby ensuring the desired level of performance. In addition, both have large internal memories and offer 
an additional removable memory capability. Another point further complicating the decision was the fact that the two have relatively similar prices, ranging from 2500 to 3500 Canadian dollars.

The final choice was the Dell Latitude 7212 Rugged Extreme. The reason was simply a matter of availability. The Panasonic tablet is more difficult to obtain, and a contact at Dell facilitated communications. The final price for the tablet was 3500 Canadian dollars, including a scanner module and an interchangeable battery.

\subsection{Designing the mobile application}

The application design required the most effort among all aspects of the project. As a result, not all of the originally planned functionalities were included in the final solution. Four functionalities were initially selected in relation to the current context and needs: Daily job site report, time sheets for workers, overtime worksheets, and access to PDF drawings. The application was designed in three complementary and iterative phases, namely, Balsamiq mock-ups, a first development phase with Qt Creator, and a second design phase with Delphi.

Mock-ups can be defined as sketches of possible user interfaces (UIs) for an application, aimed at helping stakeholders agree on different aspects of the UI (Rivero et al., 2010). Mock-ups represent the most effective solution for communicating with programmers. In the project reported in this paper, the mock-ups were designed using Balsamiq, which is a rapid prototyping tool and is well known in the field of programming (Galitz, 1993). Mock-ups show a desired result and allow programmers to follow these drawings as plans.

The first version of the platform was designed using Qt Creator. The first version of the application, based as much as possible on the provided mock-ups, could then be developed. Qt Creator is a cross-platform software development environment that integrates $\mathrm{C}++$, JavaScript, its own modeling language (QML, a derivative of HTML that allows for mobile-friendly applications), and the Qt project's libraries (Rischpater, 2013). The development of the first version of the application with Qt Creator was realized during the summer of 2018. By the end of August, an advanced interface of the first version of the application was visible. Although the interface was not fully developed at that point, the main module was ready. The interface was created for use with a keyboard and mouse, without a touchscreen. The project team evaluated the results, but was unsatisfied with the result. Thus, the first interface was abandoned.

A second attempt was made with Qt Creator (Fig. 3) using QML. However, only one person in the team had knowledge of this language. This situation caused a difficult and time-consuming development, and the application developed with Qt Creator was again decided to be abandoned.

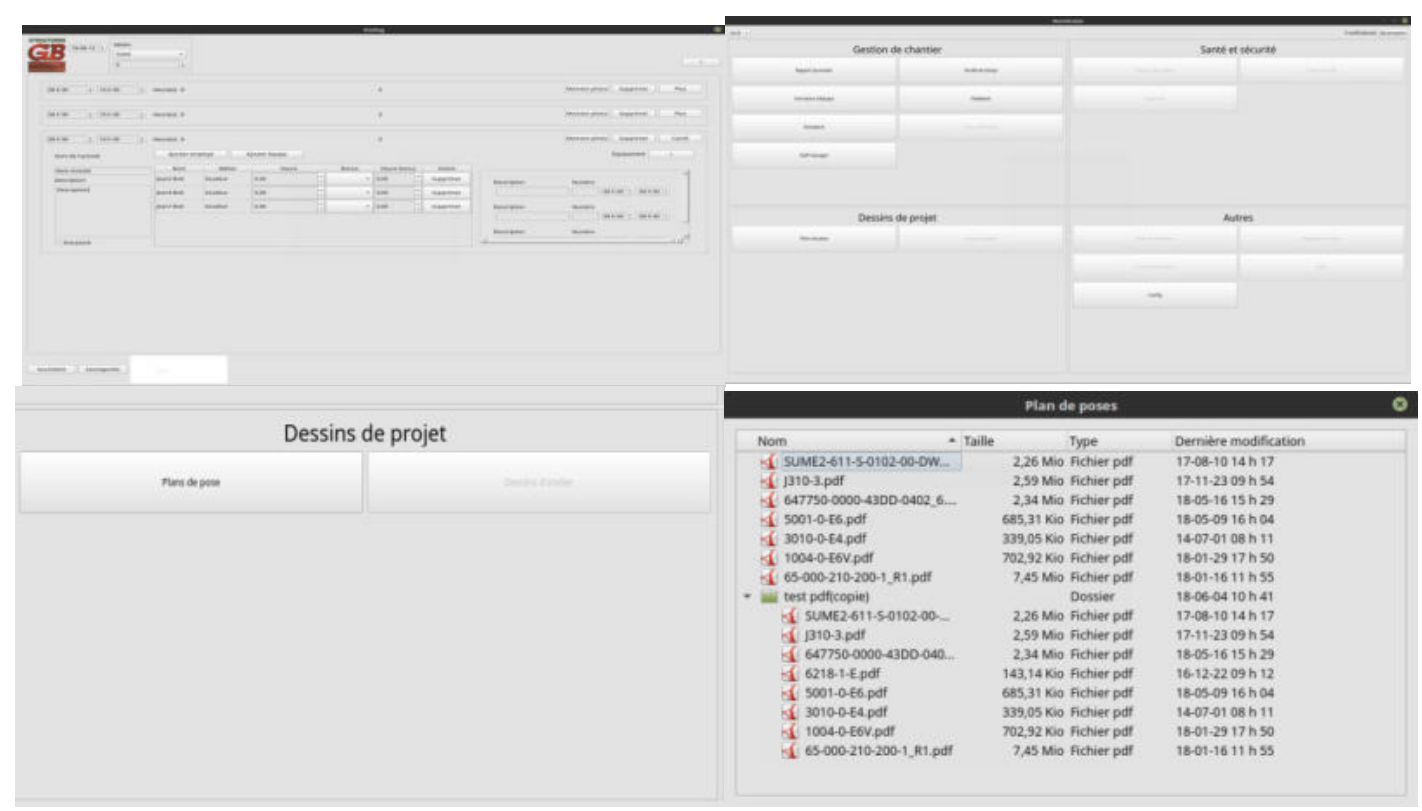

Fig. 3 Interface of the first version of the application created with Qt Creator. 
After the failure of the first version of the application created with Qt Creator, a decision was made to use the program with which most of the company's platforms are made, namely, Delphi. The team has advanced knowledge of Delphi; hence, the development of the platform could be faster. In addition, the scope of the project was limited to the daily site report requirements to have a stable application in a timely fashion. The development with Delphi lasted from October to November, 2018.

The development was conducted based on the UML class diagram presented in Fig. 4. It shows the data management process in the application. When the application is launched, the main menu is displayed. Using the three-line symbol on the top left corner as displayed in Fig. 5, the user can navigate through different pages of the application. Back on the team composition page, several teams can be created simply by selecting the names of the people who will compose the teams, proceeding by column. These teams will be retained in memory, so the user can quickly enter the team associated with an activity in the daily report. A person can also be removed from or added to an activity in the daily report. Teams were composed in this fashion because the company's site employee database can easily be linked to the application. Consequently, the human resources database is automatically updated when new employees are added.

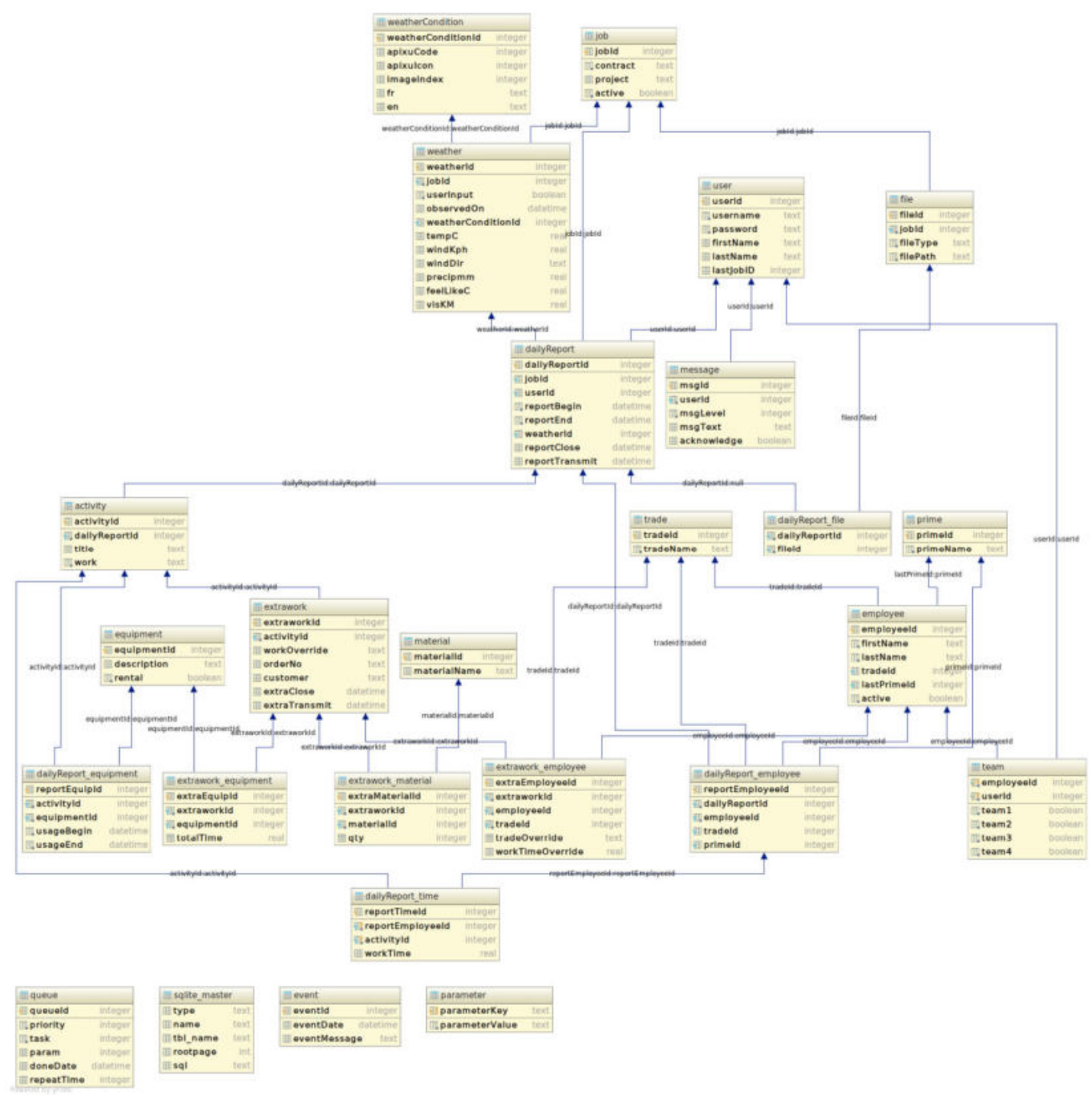

Fig. 4 UML class diagram of the data management process in the application.

When a report is created, it automatically appears in the opening menu of the daily reports. Here, the date of the report is shown, along with the number of total hours worked for the day, the report status, and the user who created it. The report can be modified or deleted. If a user attempts to delete it, a warning is shown, prompting the user to accept or cancel the deletion. When entering 
the daily report interface, the first thing the user will see is the main menu. All reports are shown here, and options are provided on the left to create a new report or to edit or delete the selected one. The user can also navigate through time to see past reports. Figure 5 shows how the site report interface is organized.

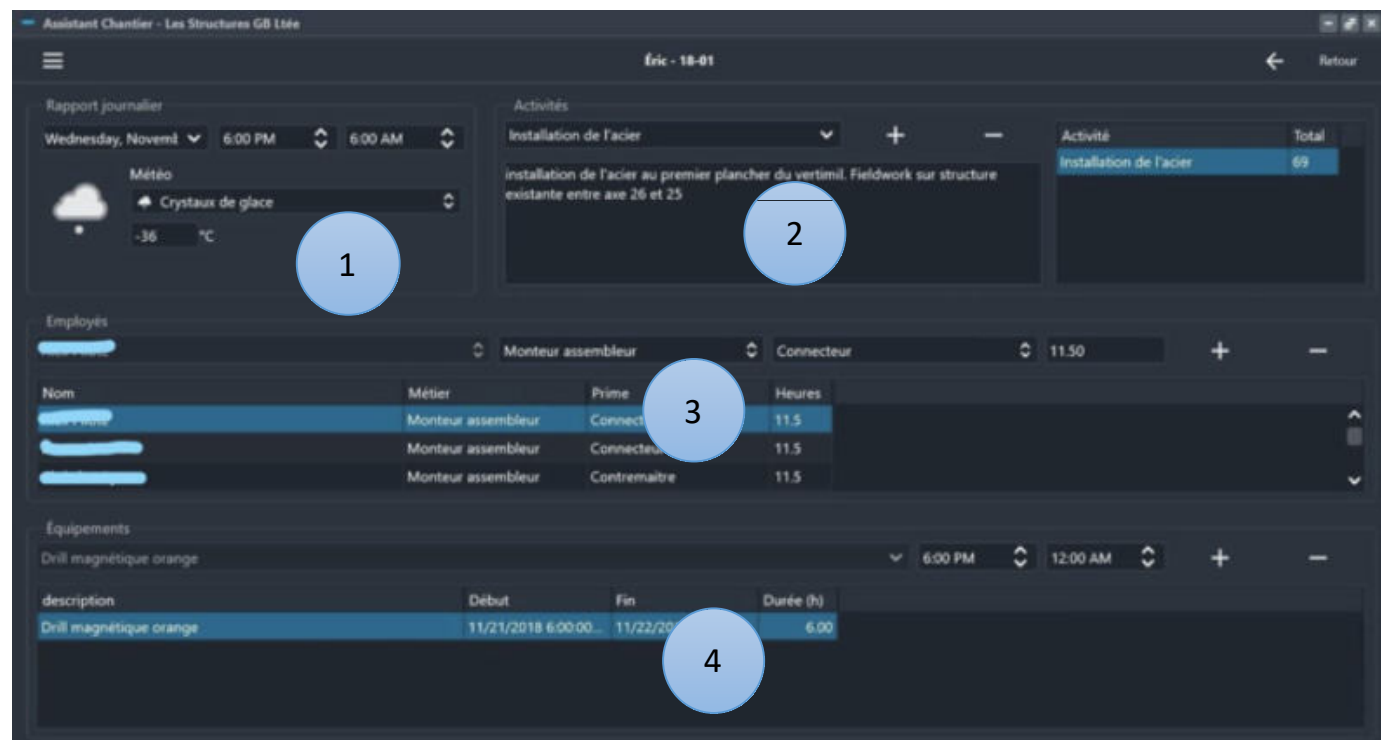

Fig. 5 Daily site report interface.

Section 1 identified in the daily report allows the user to enter the date and the time. Here, the user can also obtain information about the weather because it can greatly affect the team's performance. Section 2 summarizes the activities conducted during the work day. Each activity must be entered as a separate item in the application. On the far right, a summary of activities is found, along with associated hours. Section 3 allows the workers to author the daily report, including the daily activities and the worked hours. This step allows having the right people and the right hours for the overtime worksheets (extra work). In addition, the job of each worker is indicated, as well as whether a premium (bonus) is attached to the work performed (e.g., for the group leaders). Section 4 allows the user to $\log$ all the equipment that was used during the day. This section is important for extra work. Thus, the time slot during which a piece of equipment was used can be indicated.

\subsection{Implementing the technology: A pilot project}

\subsubsection{Project context}

The project selected for the evaluation of the proposed technology is a heavy industrial project. The site is at the northern tip of Canada, characterized by particularly cold weather. It is a 1 billion Canadian dollar project, consisting of an underground, open-pit gold mine. The mill is scheduled to go into operation in 2019. In 2017, the industrial partner erected the "Process Plant" (gold mining building) and the "Power Plant" (building housing diesel generators for power generation) at the site. In 2018, the "Crusher" (crumbling rock building), the "Crusher Dump Station" (the rock dumping building), the "Paste Plant" (building for producing concrete paste) and the "Paste Plant Dump Station" (building for rock dumping) were also erected.

\subsubsection{Evaluation of the device's durability and resistance}

Several important factors influence the choice of the selected tablet, and its durability and resistance are two of the most important ones. Theoretically, it is supposed to perform very well, to be very durable, to offer good water and dust resistance, and to be able to operate at temperatures as low as $-29^{\circ} \mathrm{C}$ (Appendix A). To experimentally verify these features, the tablet was taken to the construction site in November 2018 (Fig. 6). For the first test, it was exposed to inclement weather for $20 \mathrm{~min}$ and dropped into the snow a few times. This procedure exerted no immediate effect on the device. 


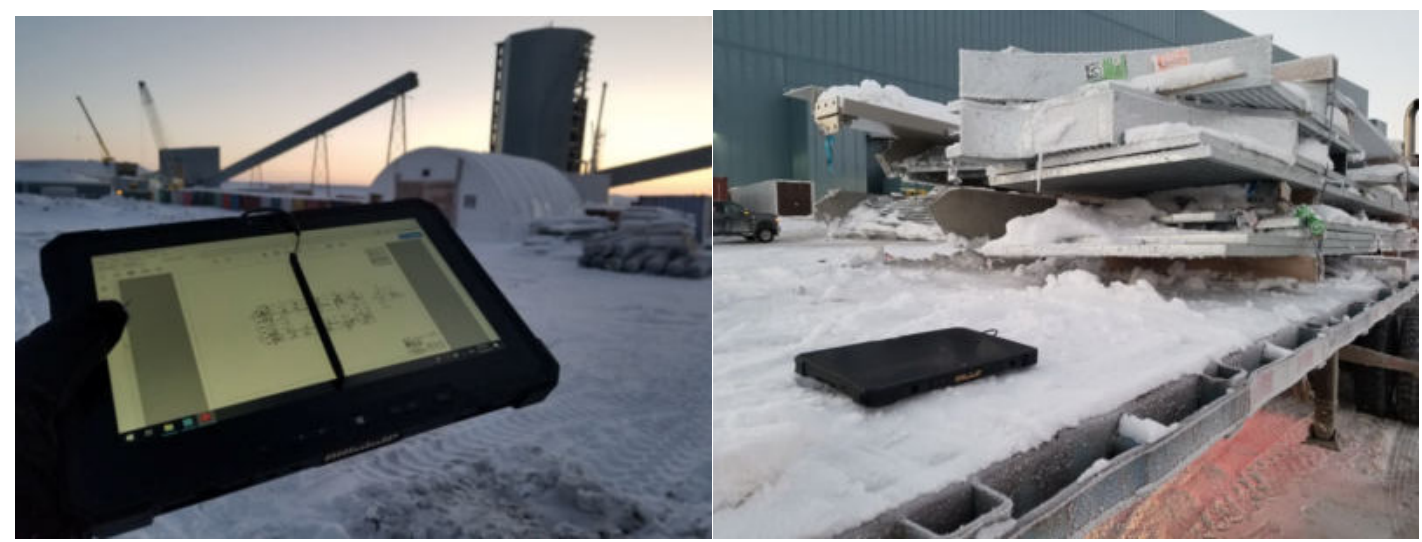

Fig. 6 Tablet operated in a $-32{ }^{\circ} \mathrm{C}$ environment.

Although 20 min of exposure may seem rather short, the temperature was as low as $-32{ }^{\circ} \mathrm{C}$. The tablet still continued to operate despite this extreme cold temperature. However, the screen and the system did not act normally, the reaction time was longer, and the sensitivity of the touchscreen was slightly affected. These observations suggest that the tablet, if used adequately, can withstand the Quebec winter.

\subsubsection{Evaluation of the application}

The application was tested in the framework of the construction site, and the foreman's feedback and recommendations were collected. The interface of the application has been considered as "correct, clear and easy to understand" by the foreman. However, he highlighted some limitations of the current version of the application and made recommendations for improvement.

The first recommendation was to have a keyboard for report drafting when the user is not on the construction site. In specific, he suggested the use of a portable Bluetooth keyboard to facilitate report writing, "because the one we have to look for in the application options is not user-friendly", he said. Second, he recommended having the application offer the possibility of quick notes, such as post-its. Currently the notes are taken in a book or on loose sheets, and the information can be easily lost under these conditions. Given that the information would already be in the tablet, transcription duplication would be avoided simply by copying and pasting the information entered in the daily report.

He also recommended an integrated library in the daily site reports, providing key phrases to simplify the entry of descriptions. The main rationale for that was that in the absence of this, the other forepersons have to dedicate a considerable amount of time in writing some description phrases, which takes us back to one of the problems raised for paper-based daily reports. He then proposed some examples of phrases: Put iron angle of border, bolting between axes $x$ and $y$, etc.

For the last recommendation, he proposed having notifications to monitor the erection work. This step would allow the foremen a better communication in addition to the daily reports that will be available for all. For example, a notification of the remaining jobs could be provided for the foreman on the next shift.

\section{Discussion and lessons learned}

The results presented above show that many challenges have to be addressed before a mobile technology aligned with the construction site requirements can be proposed. Compared to recent similar works, this research shows that BIM is a good catalyst for a paradigm change in the industry, but it is far from being reality in every construction firm. Moreover, some companies, such as the industrial partner involved in this project, have a strong preference for simple and in-house developed solutions despite the existence of cheap and powerful alternatives offered by major software companies. One question that could arise from the difficulties encountered during the application development phases is whether this decision is worthwhile. The answer, from the perspective of the industrial partner, was clearly positive. This finding can be explained not only by their desire to have internal control over their project data, but also by a need to consider 
certain specificities related to their business processes. In other words, the industrial partner prefers to invest more resources and have tailor-made tools adapted to their current practices rather than to adapt these processes to generic tools.

From the researchers' perspective, such an affirmative answer from the partner seems, at first sight, curious. Several versions of the application had to be abandoned before arriving at a final version, which, in the end, considers only a small part of the needs initially expressed by the industrial partner. Thus, understanding how a typical medium-sized contractor would prefer in-house solution, encompassing all these difficulties related to its development rather than commercial software or applications, is hard. In general, in-house solutions make sense for large architecture, engineering, and construction companies operating in complex environments with specific processes and needs, for which commercial solutions are unsuitable. For standardized small- and medium-sized projects, commercial applications are the solution in most of the cases. Obviously, the research partner involved in the project presented in this paper does not consider itself as working on standardized projects because of the nature of its construction activities. In addition, an important issue for the industrial partner is litigation management. To completely understand this issue, we must remember that this is a subcontractor who needs to document the activities carried out under these projects, especially the day-to-day activities, while keeping absolute control over this data, which can be crucial in the event of a dispute. This aspect seems to have played an important role in the preference given to a home-made solution.

Another aspect that deserves mention is the highly iterative nature of the application development process here. Two development platforms corresponding to two versions of the application had to be abandoned before we could arrive at a version that satisfied the firm. This was the case even though the mock-ups had been validated at the beginning of the process. This case illustrates some technology development issues, and also the difficulty for the partner to express their expectations clearly before a solution is available to be evaluated. This process is somehow reminiscent of the construction process, where the client's needs are unclear at the design phase, causing reworks during the construction phase. The pivotal role of the researcher, between the practitioners and the developers' team, was very important, because far from being a simple external observer, he was a stakeholder in the process. His significant contribution to the success of the project, as he understood the business needs and performed an evaluation of different proposals, suggests that action research is really adapted to this type of project. Finally, the need to function in extreme climate conditions was a crucial parameter in the choice of solutions and illustrated the peculiarity of construction sites as compared with other industries.

The needs for mobile computing solution expressed by the industrial partner teams are not unique. They correspond to the needs of most small- and medium-size enterprises (SMEs) in construction and are well documented in the scientific literature. However, the situation also concerns large construction companies and appears to be in the same forms, as shown by the research presented by Löfgren (2007). One can therefore reasonably ask the question of the utility of using home-made technologies in a general scope. Before providing generalizable answers, the factors influencing the implementation of mobile technology in construction companies must be enumerated. These factors can be summarized as follows: The complexity and cost of developing mobile application, the need to focus on the users' requirements, the need for integration with existing applications, the adaptation of content to fit multiple device types, and the choice of wireless technologies (Chen and Kamara, 2011). The factors show that while business needs can be expressed in the same way in SMEs and in large construction firms, not all of them are in the same boat. While some of the factors may nowadays be of substantially equal importance for both business categories (e.g., adaptation of content to fit multiple device types), some other factors may become rapidly critical for SMEs, such as the cost of development, the need to focus on complex users' requirements, or the availability of an internal competent development team. In this case, SMEs would therefore be more interested in opting for generic solutions available from software vendors. Large companies would have less trouble using home-made solutions but could also choose generic solutions for some specific needs.

Compared to other similar works, the present research is more comprehensive, considers the perspective of the industrial partner in the choice of the technology, and covers all the integration steps: The needs, the solution development, and the validation. Moreover, the proposed 
application is tailor-made and developed for the specific needs of the industrial partner, while the prevailing discourse currently advocates systematically the use of commercial solutions in smalland medium-sized construction companies.

\section{Conclusion and future work}

The research reported in the present paper provides a new perspective of the difficulties construction firms can face as they move to implement information technologies on their construction sites. Although many stable commercial applications exist, some firms prefer to rely on in-house competencies to develop their own applications. The study shows that developing such applications is not without difficulties, but with the need to have multiple iterations and versions before achieving the expected goals. In the case studied in this research, the initial needs evolved considerably throughout the application development and evaluation process. Finally, the project had to focus on only a part of the initial needs and to postpone the others for future work.

The project also illustrates the constraints related to the use of mobile devices on construction sites. Thus, the industrial partner had to choose tablets that could withstand extreme temperatures. The evaluation of the selected tablet was an important aspect of the process. Finally, the project shows the importance of ethnographic action research as the specific approach, because this research would not be effectively conducted using any other approach. The involvement of the researcher in the industrial partner's team throughout the duration of the project was crucial and decisive for the quality of the results obtained.

An important limitation of the research is related to the fact that the chosen solutions were evaluated only on one project and over a short period of time. Future works will be devoted to a broader assessment with projects of different characteristics, to allow inclusive and generalizable conclusions.

\section{References}

Abolfazli S, Sanaei Z, Ahmed E, Gani A, Buyya R (2014). Cloud-based augmentation for mobile devices: Motivation, taxonomies, and open challenges. IEEE Communications Surveys and Tutorials, 16(1): 337-368 doi:10.1109/SURV.2013.070813.00285

Ahmad I U, Russell J S, Abou-Zeid A (1995). Information technology (IT) and integration in the construction industry. Construction Management and Economics, 13(2): 163-171 doi:10.1080/01446199500000018

Ahmed E, Gani A, Sookhak M, Hamid S H A, Xia F (2015). Application optimization in mobile cloud computing: Motivation, taxonomies, and open challenges. Journal of Network and Computer Applications, 52: 52-68 doi:10.1016/j.jnca.2015.02.003

Ahn Y H, Kwak Y H, Suk S J (2016). Contractors' transformation strategies for adopting building information modeling. Journal of Management Engineering, 32(1): 05015005 doi:10.1061/(ASCE)ME.1943-5479.0000390

Alashwal A M, Fong P S W (2015). Empirical study to determine fragmentation of construction projects. Journal of Construction Engineering and Management, 141(7): 04015016 doi:10.1061/(ASCE)CO.1943-7862.0000986

Alsafouri S, Ayer S K (2018). Review of ICT implementations for facilitating information flow between virtual models and construction project sites. Automation in Construction, 86: 176-189 doi:10.1016/j.autcon.2017.10.005

Anumba C J, Wang X Y (2012). Mobile and pervasive computing in construction: An introduction. In: Anumba C J, Wang X Y, eds. Mobile and Pervasive Computing in Construction. New Jersey: Wiley-Blackwell, 1-10

Austin S, Baldwin A, Hammond J, Murray M, Root D, Thomson D, Thorpe A (2001). Design Chains: A Handbook for Integrated Collaborative Design. Loughborough, UK: Thomas Telford

Aziz Z, Harun A, Alaboud N (2017). Mobile computing applications within construction. In: Perera S, Ingirige B, Ruikar $\mathrm{K}$, Obonyo E, eds. Advances in Construction ICT and e-Business. Abingdon, UK: Taylor \& Francis, 404

Baccarini D (1996). The concept of project complexity—A review. International Journal of Project Management, 14(4): 201-204 doi:10.1016/0263-7863(95)00093-3

Björk B C (1999). Information technology in construction-Domain definition and research issues. International Journal of Computer Integrated Design and Construction, 1(1): 1-16

Boton C (2013). Business Views Design in Service-Oriented Information Systems. Towards Adapted Multiviews for Collaborative $4 \mathrm{D} / n \mathrm{D}$ Construction Simulation. Dissertation for the Doctoral Degree. Lorraine, France: University of Lorraine (in French) 
Boton C, Kubicki S, Halin G (2013). Designing adapted visualization for collaborative 4D applications. Automation in Construction, 36: 152-167 doi:10.1016/j.autcon.2013.09.003

Boton C, Rivest L, Forgues D, Jupp J R (2018). Comparison of shipbuilding and construction industries from the product structure standpoint. International Journal of Product Lifecycle Management, 11(3): 191-220 doi:10.1504/IJPLM.2018.094714

Bowden S, Dorr A, Thorpe T, Anumba C (2006). Mobile ICT support for construction process improvement. Automation in Construction, 15(5): 664-676 doi:10.1016/j.autcon.2005.08.004

Brydon-Miller M, Greenwood D, Maguire P (2003). Editorial: Why action research? Action Research, 1(1): 9-28 doi: $10.1177 / 14767503030011002$

Chen Y, Kamara J M (2008). Using mobile computing for construction site information management. Engineering, Construction, and Architectural Management, 15(1): 7-20 doi:10.1108/09699980810842034

Chen Y, Kamara J M (2011). A framework for using mobile computing for information management on construction sites. Automation in Construction, 20(7): 776-788 doi:10.1016/j.autcon.2011.01.002

Davies R, Harty C (2013). Implementing 'site BIM': A case study of ICT innovation on a large hospital project. Automation in Construction, 30: 15-24 doi:10.1016/j.autcon.2012.11.024

Fischer M, Kunz J (2004). The scope and role of information technology in construction. Technical Report. Center for Integrated Facility Engineering, Stanford University: 1-17

Forcada N, Macarulla M, Bortolini R (2017). Using a mobile application to assess building accessibility in smart cities. In: eWork and eBusiness in Architecture, Engineering and Construction: Proceedings of the 11th European Conference on Product and Process Modelling (ECPPM 2016). Limassol, Cyprus: 277

Futcher K, Rowlinson S (1999). IT survey within the construction industry of Hong Kong. In: 8th International Conference on Durability of Building Materials and Components. 2306-2315

Galitz W O (1993). User-Interface Screen Design. Hoboken, New Jersey: John Wiley \& Sons

Hartmann T, Fischer M, Haymaker J (2009). Implementing information systems with project teams using ethnographic-Action research. Advanced Engineering Informatics, 23(1): 57-67 doi:10.1016/j.aei.2008.06.006

Hartmann T, Van Meerveld H, Vossebeld N, Adriaanse A (2012). Aligning building information model tools and construction management methods. Automation in Construction, 22: 605-613 doi:10.1016/j.autcon.2011.12.011

Hasan A, Elmualim A, Rameezdeen R, Baroudi B, Marshall A (2018). An exploratory study on the impact of mobile ICT on productivity in construction projects. Built Environment Project and Asset Management, 8(3): 320-332 doi:10.1108/BEPAM-10-2017-0080

Hasan A, Jha K N, Rameezdeen R, Ahn S, Baroudi B (2019). Perceived productivity effects of mobile ICT in construction projects. In: Mutis I, Hartmann T, eds. Advances in Informatics and Computing in Civil and Construction Engineering. Berlin: Springer International Publishing, 165-172

Howard H, Levitt R, Paulson B C, Pohl J G, Tatum C B (1989). Computer integration: Reducing fragmentation in AEC industry. Journal of Computing in Civil Engineering, 3(1): 18-32 doi:10.1061/(ASCE)0887-3801(1989)3:1(18)

Howard R, Kiviniemi A, Samuelson O (1998). Surveys of IT in the construction industry and experience of the IT barometer in Scandinavia. Journal of Information Technology in Construction (ITcon), 3: 45-56

Jung W, Lee G (2015). The status of BIM adoption on six continents. International Journal of Civil, Environmental, Structural, Construction and Architectural Engineering, 9(5): 406-410

Kassem M, Succar B (2017). Macro BIM adoption: Comparative market analysis. Automation in Construction, 81: 286-299 doi:10.1016/j.autcon.2017.04.005

Kimoto K, Endo K, Iwashita S, Fujiwara M (2005). The application of PDA as mobile computing system on construction management. Automation in Construction, 14(4): 500-511 doi:10.1016/j.autcon.2004.09.003

Kubicki S (2006). Assisting Flexible Coordination in Building Construction Activity: A Model-Based Approach to Develop New Tools for Cooperation Context Vizualisation. Dissertation for the Doctoral Degree. Nancy, France: Université Henri Poincaré, Nancy I (in French)

Kubicki S, Boton C (2014). IT barometer survey in Luxembourg: First results to understand IT innovation in construction sector. In: Cohn L F, ed. Computing in Civil and Building Engineering. New York: American Society of Civil Engineers, $179-186$

Löfgren A (2007). Mobility in-site: Implementing mobile computing in a construction enterprise. Communications of the Association for Information Systems, (20): 594-604

Marsh L, Flanagan R (2000). Measuring the costs and benefits of information technology in construction. Engineering, Construction, and Architectural Management, 7(4): 423-435 doi:10.1046/j.1365-232x.2000.00177.x

Neufert E (2002). Elements of Construction Projects. Paris: Dunod (in French)

Omar T, Nehdi M L (2016). Data acquisition technologies for construction progress tracking. Automation in Construction, 70: $143-155$ doi:10.1016/j.autcon.2016.06.016 
Orihuela P, Orihuela J, Pacheco S (2016). Information and communications technology in construction: A proposal for production control. Procedia Engineering, 164: 150-157 doi:10.1016/j.proeng.2016.11.604

Park J, Cho Y K, Kim K (2016). Field construction management application through mobile BIM and location tracking technology. In: Proceedings of the 33rd International Symposium on Automation and Robotics in Construction. Auburn, AL: 83-89

Patrick C (2003). Construction Project Planning and Scheduling. Englewood, New Jersey: Prentice Hall

Rebolj D, Menzel K (2004). Mobile computing in construction. Journal of Information Technology in Construction (ITcon), 9(19): 281-283

Rischpater R (2013). Application Development with Qt Creator. Birmingham: Packt Publishing

Rivard H (2000). A survey on the impact of information technology on the Canadian architecture, engineering and construction industry. Journal of Information Technology in Construction (ITcon), 5: 37-56

Rivero J M, Rossi G, Grigera J, Burella J, Luna E R, Gordillo S (2010). From mockups to user interface models: An extensible model driven approach. In: International Conference on Web Engineering. Berlin, Heidelberg: Springer Verlag, $13-24$

Roy M, Prévost P (2013). Action research: Origins, characteristics and implications of its use in the management sciences. Qualitative Research, 32(2): 129-151 (in French)

Sacks R, Eastman C, Lee G, Teicholz P (2018). BIM Handbook: A Guide to Building Information Modeling for Owners, Designers, Engineers, Contractors, and Facility Managers. Hoboken, New Jersey: Wiley

Samuelson O (2008). The IT-barometer-A decade's development of IT use in the Swedish construction sector. Journal of Information Technology in Construction (ITcon), 13: 1-19

Samuelson O, Björk B C (2014). A longitudinal study of the adoption of IT technology in the Swedish building sector. Automation in Construction, 37: 182-190 doi:10.1016/j.autcon.2013.10.006

Silverio M, Renukappa S, Suresh S, Donastorg A (2017). Mobile computing in the construction industry: Main challenges and solutions. In: Benlamri R, Sparer M, eds. Leadership, Innovation and Entrepreneurship as Driving Forces of the Global Economy. Cham: Springer, 85-99

Sunke N (2009). Planning of Construction Projects: A Managerial Approach. Dissertation for the Doctoral Degree. Siegen, Germany: University of Siegen

Svalestuen F, Knotten V, Lædre O, Drevland F, Lohne J (2017). Using building information model (BIM) devices to improve information flow and collaboration on construction sites. Journal of Information Technology in Construction (ITcon), 22: 204-219

Turk Ž (2016). Ten questions concerning building information modelling. Building and Environment, 107: 274-284 doi:10.1016/j.buildenv.2016.08.001

Yeh K C, Tsai M H, Kang S C (2012). On-site building information retrieval by using projection-based augmented reality. Journal of Computing in Civil Engineering, 26(3): 342-355 doi:10.1061/(ASCE)CP.1943-5487.0000156 